Dossier: Mujeres y Educación en la Universidad Nacional

Red de Mujeres Investigadoras de la Universidad Nacional (UNA)

\title{
Perfil de la graduada de la carrera de Administración y Gestión de Recursos Humanos de la UTN
}

\author{
Kattia Lizzett Vasconcelos Vásquez \\ Universidad Técnica Nacional, Costa Rica \\ kvasconcelos@utn.ac.cr \\ http://orcid.org/0000-0001-6803-4360 \\ Betzaida Montero Ulate \\ Universidad Técnica Nacional, Costa Rica \\ bmontero@utn.ac.cr \\ http://orcid.org/0000-0002-9574-4584
}

Recibido: 4 de agosto de 2020

Aceptado: 10 de noviembre de 2020

Resumen: Este documento es parte de una investigación mayor cuyo objetivo es conocer las características del perfil profesional de la graduada de la carrera de Administración y Gestión de Recursos Humanos de la Universidad Técnica Nacional. Su intención es visibilizar que la carrera mencionada es una opción viable para las mujeres que optan por continuar sus estudios universitarios según la oferta académica de la universidad, de acuerdo con las preferencias de las estudiantes de la provincia de Alajuela y sus cantones. Los resultados de este estudio se obtienen a partir de una población de 85 graduadas de la carrera AGRH durante el período 2012-2019.

Palabras clave: Educación superior; género; recursos humanos; talento humano.

\section{(ब) $(\Theta \odot$}

La Revista Estudios es editada por la Universidad de Costa Rica y se distribuye bajo una Licencia Creative Commons Atribución-NoComercial-CompartirIgual 3.0 Costa Rica. Para más información envíe un mensaje a revistaestudios.eeg@ucr.ac.cr. 
Dossier: Mujeres y Educación en la Universidad Nacional

Red de Mujeres Investigadoras de la Universidad Nacional (UNA)

\title{
Profile of the graduate of the career of administration and management of human resources of la UTN
}

\begin{abstract}
This document is part of a larger investigation whose objective is to know the characteristics of the professional profile of the graduate of the Administration and Management of Human Resources career at the National Technical University, with the intention of making visible that the aforementioned career is an education option. superior for women according to the academic offer of the University and preferably for students from the province of Alajuela and its cantons. The results of this study are obtained from a population of 85 graduates of the AGRH degree in the period 2012-2019.
\end{abstract}

Keywords: Gender; Higher education; Human Resources; Human talent.

\section{Introducción}

La carrera universitaria de Administración y Gestión de Recursos Humanos cuenta con 11 años de oferta académica y surge a partir de la constitución de la Universidad Técnica Nacional de ahora en adelanta UTN, que a su vez fue creada con el propósito de atender las necesidades de formación técnica que requiere el país en todos los niveles de educación superior, según se establece en la Ley No. 8638 en su artículo 1. La constitución de esta institución estatal de educación superior universitaria queda en firme a partir de la firma de la ley mencionada por la Asamblea Legislativa en ejercicio y publicada en el Alcance No. 22-A a La Gaceta No.107. La Ley No. 8638 se ratifica por el Poder Ejecutivo el 4 de junio del 2008, lo cual oficializó la creación del centro educativo.

\section{(ब) $\odot \odot$}

La Revista Estudios es editada por la Universidad de Costa Rica y se distribuye bajo una Licencia Creative Commons Atribución-NoComercial-CompartirIgual 3.0 Costa Rica. Para más información envíe un mensaje a 
Dossier: Mujeres y Educación en la Universidad Nacional

Red de Mujeres Investigadoras de la Universidad Nacional (UNA)

La UTN nace de la fusión de varios colegios universitarios públicos según se establece en la ley de creación en su artículo 7:

a. El Colegio Universitario de Alajuela, (CUNA), creado según Ley No. 6541, el 19 de noviembre de 1980. Donde originalmente se daba el diplomado de recursos humanos.

b. El Centro de Investigación y Perfeccionamiento de la Enseñanza Técnica (CIPET), establecido por el artículo 70 de la Ley No. 6995, del 22 de julio de 1985, y regulado por el Decreto Ejecutivo No. 21167-MEP, del 17 de marzo de 1992.

c. El Centro de Formación de Formadores y Personal Técnico para el Desarrollo Industrial de Centroamérica (CEFOF), creado por Decreto Ejecutivo No. 21331MEP, de 2 de julio de 1992, y regulado por Decreto Ejecutivo No. 31529-MPRMICITT, del 13 de marzo del 2003.

d. La Escuela Centroamericana de Ganadería (ECAG), creada por Ley No. 4401, del 1 de septiembre de 1969.

e. El Colegio Universitario de Puntarenas (CUP), creado según Ley No. 6541, del 19 de noviembre de 1980.

f. El Colegio Universitario para el Riego y Desarrollo del Trópico Seco (CURDTS), creado según Ley No. 7403, del 3 de mayo de 1994.

A su vez, también se constituye una Comisión de Conformación de la UTN, comisión ad-hoc, que tiene a su cargo las funciones de gobierno, administración y dirección. Para el año 2009 la UTN inicia lecciones mediante la apertura de 33 carreras de diplomado en sus diferentes sedes, entre ellas la carrera de Administración y Gestión de Recursos Humanos, de ahora en adelante AGRH.

\section{(c) (i) (2)}

La Revista Estudios es editada por la Universidad de Costa Rica y se distribuye bajo una Licencia Creative Commons Atribución-NoComercial-CompartirIgual 3.0 Costa Rica. Para más información envíe un mensaje a revistaestudios.eeg@ucr.ac.cr. 
Dossier: Mujeres y Educación en la Universidad Nacional

Red de Mujeres Investigadoras de la Universidad Nacional (UNA)

Esta carrera se formaliza a partir de la Comisión de Conformación de la UTN, quien recibe y aprueba el dictamen remitido por la Oficina de Planificación de la Educación Superior (OPES), sobre el programa de estudios de la carrera de Diplomado en Administración de Recursos Humanos, en la sesión 17-2008, celebrada el 15 de diciembre de 2008.

La carrera oferta el primer ciclo en el primer cuatrimestre del 2009, esperando contar con la primera promoción de graduados que complete el plan de estudios para el tercer cuatrimestre del 2010, la cual se rige por la Política de Admisión de la UTN en su artículo 1 que dice:

El mérito académico, medido por el promedio de notas de Educación Diversificada, o su equivalente, es el principal criterio para la selección de estudiantes en su ingreso a la UTN, sin demérito del acceso especial de poblaciones vulnerables por factores de tipo socioeconómico y de oportunidades educativas.

Asimismo en el artículo 2 se menciona:

De conformidad con lo anterior, las siguientes poblaciones son prioritarias para el ingreso a la UTN:

a) Graduados de colegios públicos de los 24 cantones de menor índice de desarrollo humano, según el último estudio publicado por el PNUD.

b) Población trabajadora.

c) Graduados de Colegios Técnicos Profesionales públicos y semipúblicos que hayan cursado especialidades afines a la carrera que deseen matricular en la UTN.

d) Graduados del Instituto Nacional de Aprendizaje (INA) de especialidades afines a la carrera que deseen matricular en la UTN.

e) Graduados de colegios públicos que cursaron durante el cuarto y quinto año, una tecnología afín a la carrera que deseen matricular en la UTN.

\section{(c) (i) (2) (2)}

La Revista Estudios es editada por la Universidad de Costa Rica y se distribuye bajo una Licencia Creative Commons Atribución-NoComercial-CompartirIgual 3.0 Costa Rica. Para más información envíe un mensaje a 
Dossier: Mujeres y Educación en la Universidad Nacional

Red de Mujeres Investigadoras de la Universidad Nacional (UNA)

f) Graduados de los Programas de Técnico Universitario Superior que imparte la UTN, en especialidades afines a la carrera que deseen matricular.

El perfil académico profesional que certifica la UTN mediante el título de Bachiller en Administración y Gestión de Recursos Humanos y que se contempla en el Plan de Estudios en el capítulo V denominado Campo de Inserción Laboral (2011) indica:

El conocimiento, habilidades y destrezas adquiridas en el proceso de formación que brinda el programa de este bachillerato, abre las posibilidades de inserción en los tres grandes campos de la actividad profesional, sea en la investigación, la docencia o el ejercicio liberal subdividiéndose este en la consultoría o asesoría a las organizaciones, a nivel ejecutor de los procesos de gestión de recursos humanos o como responsables de los programas de gestión que en el campo tienen todas las organizaciones (p.28).

De acuerdo con los campos de inserción laboral para la graduada de la carrera AGRH según su perfil profesional, Guédez (1980) citado en Núñez y Marín (2018), indican que el perfil profesional de una determinada carrera universitaria se define como:

El conjunto de características pretendidas por el empleador, las cuales deben reflejar las exigencias del mercado ocupacional, en términos de los requisitos que definan las habilidades, las destrezas, los rasgos de personalidad, la conformación física y el nivel de educación inherente al desempeño profesional. (pp. 5-6).

En relación con la anterior, Díaz (1995), Arnaz (1981), citado en Damián y Arellando (2009), existen definiciones de perfil profesional que coinciden según ciertos elementos, por tanto, es el conjunto de capacidades y competencias que identifican la formación de una persona para asumir en condiciones óptimas las responsabilidades propias del desarrollo de funciones y tareas de una determinada profesión (pp. 7-8). A partir del registro de graduaciones en el período 2012-2019,

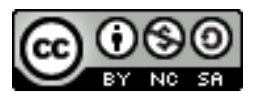

La Revista Estudios es editada por la Universidad de Costa Rica y se distribuye bajo una Licencia Creative Commons Atribución-NoComercial-CompartirIgual 3.0 Costa Rica. Para más información envíe un mensaje a revistaestudios.eeg@ucr.ac.cr. 
Dossier: Mujeres y Educación en la Universidad Nacional

Red de Mujeres Investigadoras de la Universidad Nacional (UNA)

se cuenta con un total de 503 profesionales que obtienen el título de bachiller universitario en la carrera, de las cuales 409 son mujeres.

La carrera se enmarca en el área de Ciencias Económicas y la disciplina de Administración de Recursos Humanos, según la nomenclatura establecida por el Consejo Nacional de Rectores (CONARE) y la Oficina de Planificación de la Educación Superior (OPES). Ambos organismos establecen un formato para organizar la oferta de carreras universitarias impartidas en el país basado en los campos del conocimiento de la Organización de las Naciones Unidas para la Educación, la Ciencia y Cultura (UNESCO).

La carrera tiene presencia en la Sede Central y la Sede San Carlos. Actualmente se encuentra en proceso de autoevaluación para optar por la acreditación a través del Sistema Nacional de Acreditación de la Educación Superior (SINAES). Este propósito es liderado por la Comisión General de la Carrera AGRH InterSedes que inicia labores el 10 de marzo del 2016. El proceso de autoevaluación implica la consecución de acciones que llevan a la carrera a optimizar procesos internos relacionados al cumplimiento de metas operativas, planes de investigación y extensión, desarrollo académico/docente, rediseño del plan de estudios, entre otros.

La mejora continua es la consigna para mantener una carrera acreditada. Por ello desde la dirección de carrera se generan sinergias entre diversos actores, en coordinación con la Comisión General de la Carrera y la Dirección de Evaluación Académica. Para esto se convoca a estudiantes, egresados, empleadores y docentes a conformarse en equipos de trabajo consultivos que permitan una mayor claridad sobre el perfil de salida, en contraposición con las expectativas, exigencias y necesidades del mercado laboral actual.

\section{(c) (i) (2)}

La Revista Estudios es editada por la Universidad de Costa Rica y se distribuye bajo una Licencia Creative Commons Atribución-NoComercial-CompartirIgual 3.0 Costa Rica. Para más información envíe un mensaje a 
Dossier: Mujeres y Educación en la Universidad Nacional

Red de Mujeres Investigadoras de la Universidad Nacional (UNA)

La carrera $A G R H$ se ha planteado en los últimos meses impulsar espacios que permitan a sus académicos desarrollar propuestas producto de diferentes evidencias y logros obtenidos en el devenir de los cursos. De esta forma, se busca motivar líneas de investigación que apalanquen la producción académica en conjunto con la articulación de acciones de extensión con el sector socio productivo, variable de éxito para dar continuidad a una futura etapa de reacreditación.

Según los planes de estudios que procura el mercado de universidades públicas y privadas, la UTN brinda la modalidad cuatrimestral y es la única que ofrece un diplomado/pregrado en administración de recursos humanos. El plan de estudios para el diplomado se compone de seis niveles ( 6 cuatrimestres), para un total de 72 créditos y otorga la salida lateral con un diplomado en Administración de Recursos Humanos. El bachillerato/grado lo conforma 10 niveles (10 cuatrimestres), y está constituido por los 6 niveles del diplomado más 4 niveles adicionales para un total de 128 créditos.

Es relevante visibilizar que la carrera $A G R H$ es la opción de educación superior acogida por mujeres según la oferta académica de la UTN y de preferencia para la población femenina de la provincia de Alajuela y sus cantones.

Para el período 2012-2019, el $81 \%$ de los graduados de la carrera son femíneas, y para el primer cuatrimestre 2020 se cuenta con una matrícula de 488 estudiantes, de las cuales 410 son mujeres, que corresponde a un $84 \%$ de la población activa, según los datos de la oficina de Registro Universitario en la Sede Central.

Por lo anterior, se genera el objetivo general a saber: Determinar el perfil profesiográfico de las graduadas de la carrera de Administración y Gestión de

La Revista Estudios es editada por la Universidad de Costa Rica y se distribuye bajo una Licencia Creative Commons Atribución-NoComercial-CompartirIgual 3.0 Costa Rica. Para más información envíe un mensaje a revistaestudios.eeg@ucr.ac.cr. 
Dossier: Mujeres y Educación en la Universidad Nacional

Red de Mujeres Investigadoras de la Universidad Nacional (UNA)

Recursos Humanos para el establecimiento de sus características demográficas y afines durante el periodo 2012-2019. Para lograr el objetivo general se diseñan los objetivos específicos que se enuncian a continuación: Enunciar las características demográficas, preparación académica, apoyo socioeconómico y trayectoria laboral y definir el perfil profesional de la graduada AGRH para el establecimiento de la migración para obtener un título universitario.

\section{Metodología}

En esta investigación utiliza la metodología cualitativa porque se ajusta a lo indicado por Cohen N y Gómez Rojas (2019): "Su interés es reproducir la perspectiva del actor, su mirada, sus representaciones o caracterizar estadísticamente el desempeño de un colectivo o conjunto de sujetos, de países, de instituciones, etc." (p. 10).

Este tipo de metodología permite que se ajuste a las necesidades de las personas participantes como se indica en el estudio de casos como lo señala Borges (1995).

Por otra parte, el mismo Borges manifiesta que "el estudio de caso ha ido adquiriendo su personalidad literaria y epistemológica en función de su uso como instrumento pedagógico y/o como herramienta o estrategia de investigación" (1995; p.5).

También Yin, (1989) “...el estudio de caso investigativo constituye una herramienta de mayor alcance, que posibilita la generación de conocimiento y la comprensión de determinados fenómenos" (p.2). Además, Borges (1995) señala que: "buscan ampliar las posibilidades de interpretación, intentando incorporar la complejidad de la toma de decisiones, a la vez que ambas requieren de base de datos y del desarrollo de herramientas de recopilación y análisis de información" (p.2).

\section{(c) (i) (2) (2)}

La Revista Estudios es editada por la Universidad de Costa Rica y se distribuye bajo una Licencia Creative Commons Atribución-NoComercial-CompartirIgual 3.0 Costa Rica. Para más información envíe un mensaje a 
Dossier: Mujeres y Educación en la Universidad Nacional

Red de Mujeres Investigadoras de la Universidad Nacional (UNA)

Además, Borges (1995) considera los siguientes aspectos:

(1) Investiga un fenómeno contemporáneo en su contexto real.

(2) Cuando los límites entre el fenómeno y el contexto no son evidentes o claramente diferenciables.

(3) Utiliza múltiples fuentes de evidencia o datos.

(4) Utiliza primordialmente (pero no exclusivamente) métodos cualitativos de investigación (1995; p. 6)

Entonces en esta investigación se cumple con:

(1) Las graduadas de la carrera de AGRH son contemporáneas y tienen un contexto real.

(2) Se necesita hacer una valoración cualitativa entre su entorno, formación y realidad laboral.

(3) Se verifican los antecedentes como estudiantes en las bases del departamento de registro de la UTN. Se tiene una experiencia con las investigadoras en diferentes cursos de la carrera y se trabaja con un formulario en línea para que las graduadas lo completen.

(4) Es una revisión de los elementos cualitativos de las respuestas arrojadas en la base diseñada para esta investigación.

De manera que la metodología de casos se aplica adecuadamente en esta investigación. Además, para complementar este tipo de investigación se retoma a Pérez Aguilar, W. (1999), Chetty (1996), Stake (1995) o Yin (1981, 1989, 1993 y 1999). En particular considerando un estudio de casos (Yin, 1989 y 1993) y a Castro Monge (2010) que hace el aporte directo a la administración.

\section{(c) (i) (9)}

La Revista Estudios es editada por la Universidad de Costa Rica y se distribuye bajo una Licencia Creative Commons Atribución-NoComercial-CompartirIgual 3.0 Costa Rica. Para más información envíe un mensaje a 
Dossier: Mujeres y Educación en la Universidad Nacional

Red de Mujeres Investigadoras de la Universidad Nacional (UNA)

De tal forma que en el estudio de casos hay que tener en cuenta dos aspectos importantes: número de casos y criterios de selección de este (Yin, 1989). Esto va totalmente de la mano con la participación de las mujeres de la carrera de AGRH siendo una población representativa. Se gradúan de la misma carrera, con procedencia de diferentes provincias del país y de colegios similares.

Por lo que, al verificar la información los resultados en algunos casos son muy parecidos con un margen de diferencia menor.

El enfoque de esta investigación es mixto porque tiene datos como: edad, estado civil, y elementos cualitativos como opiniones de las evaluadas. La población con la que se trabaja es de 85 graduadas durante periodo 2012-2019 que estaban disponibles al completar el instrumento diseñado. La selección de esta población se hace a partir del listado facilitado por el Departamento de Registro de la UTN. Para la encuesta, se diseña un instrumento dirigido a las graduadas de la carrera AGRH a partir de los siguientes criterios:

A. Datos demográficos: considera elementos relacionados a edad, estado civil, provincia y cantón de residencia, condición casa de habitación, dependientes a cargo.

B. Preparación académica: se refiere a la continuación de estudios superiores, especialización o programas técnicos que complementen su desarrollo profesional.

C. Apoyo socioeconómico: determina el apoyo o respaldo económico a la graduada en su condición de estudiante para el ingreso, permanencia y conclusión de estudios universitarios.

\section{(cc) (iહ)ણு}

La Revista Estudios es editada por la Universidad de Costa Rica y se distribuye bajo una Licencia Creative Commons Atribución-NoComercial-CompartirIgual 3.0 Costa Rica. Para más información envíe un mensaje a 
Dossier: Mujeres y Educación en la Universidad Nacional

Red de Mujeres Investigadoras de la Universidad Nacional (UNA)

D. Trayectoria profesional: implica la ruta o trayecto de la graduada para el desarrollo de su carrera profesional.

Se valida la estructura, organización y redacción de cada pregunta entre los docentes investigadores y un grupo de cinco graduadas de diversas generaciones.

Recogida de los datos:

Se realiza por medio de una encuesta, a través de un formulario en línea. Se aplica durante el periodo del 8 de abril al 10 de mayo inclusive del 2020.

Nivel de respuesta:

De acuerdo con la población de interés, se concreta la participación y respuesta de 85 profesionales graduadas en el período 2012 - 2019.

\section{Resultados}

En este apartado se muestran los principales datos de las características demográficas, formación académica, apoyo socioeconómico y trayectoria. Entre las diferencias sociodemográficas de las mujeres graduadas de la carrera de AGRH y en el rango de edad de 24 a 27 años, se obtiene la mayor participación según se destaca en la figura 1:

\section{(c) (i)(2)}

La Revista Estudios es editada por la Universidad de Costa Rica y se distribuye bajo una Licencia Creative Commons Atribución-NoComercial-CompartirIgual 3.0 Costa Rica. Para más información envíe un mensaje a revistaestudios.eeg@ucr.ac.cr. 
Dossier: Mujeres y Educación en la Universidad Nacional

Red de Mujeres Investigadoras de la Universidad Nacional (UNA)

Figura 1

Edad de la Graduada de la carrera AGRH de la UTN

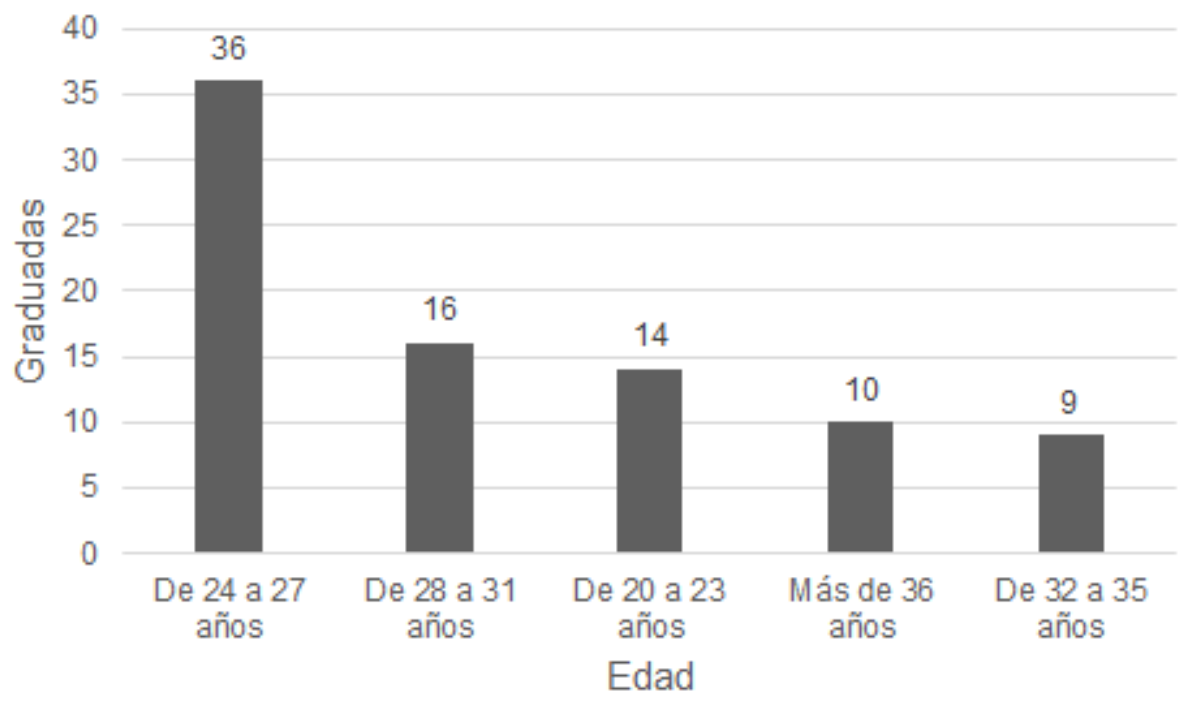

Fuente: Instrumento aplicado

Elaboración: propia.

Se considera la provincia y lugar de procedencia de la graduada, donde Alajuela y su cantón central, Alajuela, concentra la mayor cantidad de participantes, según se establece en la tabla 1:

\section{(c) (i) (2) (2)}

La Revista Estudios es editada por la Universidad de Costa Rica y se distribuye bajo una Licencia Creative Commons Atribución-NoComercial-CompartirIgual 3.0 Costa Rica. Para más información envíe un mensaje a revistaestudios.eeg@ucr.ac.cr. 
Dossier: Mujeres y Educación en la Universidad Nacional

Red de Mujeres Investigadoras de la Universidad Nacional (UNA)

Tabla 1

Provincia y cantón de procedencia de la graduada de la carrera AGRH de la UTN

\begin{tabular}{|l|c|l|c|}
\hline Provincia & No. Graduadas & Cantón & No. Graduadas \\
\hline Alajuela & 72 & Alajuela & 49 \\
\hline Heredia & 9 & Otros & 8 \\
\hline San José & 3 & Naranjo & 7 \\
\hline Puntarenas & 1 & Poás & 5 \\
\hline & & Grecia & 3 \\
\hline & & Heredia & \\
\hline & & Palmares & 3 \\
\hline Total & & Atenas & 2 \\
\hline & & Belén & 2 \\
\hline
\end{tabular}

Fuente: Instrumento aplicado

Elaboración: propia.

La condición de estado civil, casa de habitación, personas que viven y dependientes a cargo, completan el perfil sociodemográfico según la tabla 2.

\section{(c) (i) (9) (2)}

La Revista Estudios es editada por la Universidad de Costa Rica y se distribuye bajo una Licencia Creative Commons Atribución-NoComercial-CompartirIgual 3.0 Costa Rica. Para más información envíe un mensaje a revistaestudios.eeg@ucr.ac.cr. 
Dossier: Mujeres y Educación en la Universidad Nacional

Red de Mujeres Investigadoras de la Universidad Nacional (UNA)

Tabla 2

\begin{tabular}{|c|c|c|c|c|c|c|c|}
\hline $\begin{array}{l}\text { Estado } \\
\text { Civil }\end{array}$ & $\begin{array}{l}\text { No. } \\
\text { Graduadas }\end{array}$ & $\begin{array}{l}\text { Condición } \\
\text { casa } \\
\text { habitación }\end{array}$ & $\begin{array}{l}\text { No. } \\
\text { Graduadas }\end{array}$ & $\begin{array}{c}\text { Personas } \\
\text { por casa } \\
\text { habitación }\end{array}$ & $\begin{array}{l}\text { No. } \\
\text { Graduadas }\end{array}$ & $\begin{array}{c}\text { Dependientes } \\
\text { a cargo }\end{array}$ & $\begin{array}{c}\text { No. } \\
\text { Graduadas }\end{array}$ \\
\hline Soltera & 58 & Progenitores & 31 & $\begin{array}{l}1 \text { a } 2 \\
\text { personas }\end{array}$ & 39 & 1 persona & 41 \\
\hline Casada & 17 & Propia & 29 & $\begin{array}{l}3 \text { a } 4 \\
\text { personas }\end{array}$ & 30 & 2 personas & 19 \\
\hline Divorciada & 5 & Alquilada & 20 & $\begin{array}{l}5 \text { a } 6 \\
\text { personas }\end{array}$ & 15 & Ninguna & 15 \\
\hline \multirow[t]{2}{*}{ Unión libre } & 5 & Prestada & 5 & $\begin{array}{l}7 \text { personas o } \\
\text { más }\end{array}$ & 1 & 3 personas & 5 \\
\hline & & & & & & 4 personas & 5 \\
\hline Total & 85 & & 85 & & 85 & & 85 \\
\hline
\end{tabular}

Fuente: Instrumento aplicado

Elaboración: propia.

La preparación académica de las graduadas de la carrera AGRH se enmarca en la continuación de estudios superiores, especialización o programas técnicos que complementen su desarrollo profesional. Previo se contempla la procedencia de la graduada en términos de su educación secundaria según se observa en la tabla 3.

Tabla 3

Educación secundaria de la graduada de la carrera AGRH de la UTN

\begin{tabular}{cc}
\hline Modalidad Educativa & Cantidad \\
\hline Colegio Académico & 61 \\
Colegio Técnico Profesional & 13 \\
Bachillerato por Madurez & 7 \\
Colegio Experimental & 3 \\
Colegio Bachillerato Internacional & 1 \\
\hline Total & $\mathbf{8 5}$ \\
\hline
\end{tabular}

Fuente: Instrumento aplicado

Elaboración: propia.

\section{(c) (i)(2)}

La Revista Estudios es editada por la Universidad de Costa Rica y se distribuye bajo una Licencia Creative Commons Atribución-NoComercial-CompartirIgual 3.0 Costa Rica. Para más información envíe un mensaje a revistaestudios.eeg@ucr.ac.cr. 
Dossier: Mujeres y Educación en la Universidad Nacional

Red de Mujeres Investigadoras de la Universidad Nacional (UNA)

La figura 2 muestra la relación entre el año de ingreso y año de graduación en la carrera AGRH de la UTN, donde a partir del 2017 se establece un crecimiento significativo de graduadas que finalizan su plan de estudios:

Figura 2

Relación año de ingreso y de graduación de la carrera AGRH de la UTN

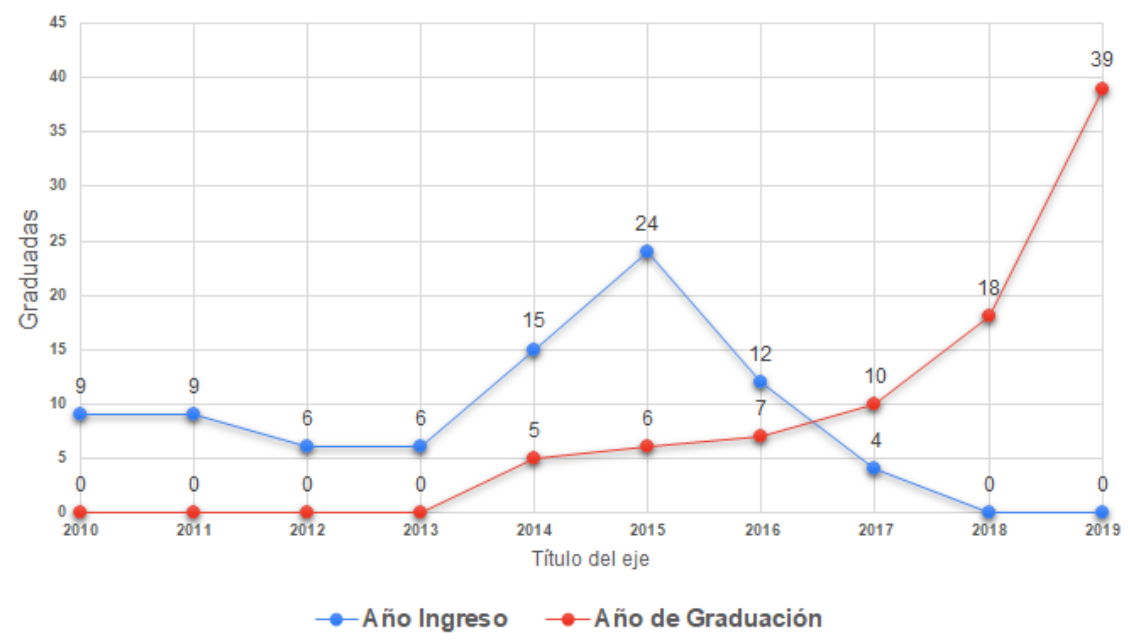

Fuente: Instrumento aplicado

Elaboración: propia.

La continuidad de estudios superiores, especialización o programas técnicos es un tema que ocupa a 30 de las 85 graduadas que participan en el estudio, de acuerdo con la información descrita en la tabla 4.

\section{(c) (i) (2) (2)}

La Revista Estudios es editada por la Universidad de Costa Rica y se distribuye bajo una Licencia Creative Commons Atribución-NoComercial-CompartirIgual 3.0 Costa Rica. Para más información envíe un mensaje a revistaestudios.eeg@ucr.ac.cr. 
Dossier: Mujeres y Educación en la Universidad Nacional

Red de Mujeres Investigadoras de la Universidad Nacional (UNA)

\section{Tabla 4}

Estudios superiores, especialización o programas técnicos de la graduada de la carrera AGRH de la UTN

\begin{tabular}{lcc}
\hline Curso/Especialización & $\begin{array}{c}\text { Frecuencia } \\
\text { absoluta }\end{array}$ & $\begin{array}{c}\text { Frecuencia } \\
\text { relativa }\end{array}$ \\
\hline Licenciatura AGRH & 11 & $34 \%$ \\
Licenciatura & 7 & $22 \%$ \\
Curso de Inglés & 3 & $9 \%$ \\
Curso contabilidad & 2 & $6 \%$ \\
Capacitación compensación & 1 & $3 \%$ \\
Carrera inglés & 1 & $3 \%$ \\
Curso relaciones personales & 1 & $3 \%$ \\
Especialización economía solidaria & 1 & $3 \%$ \\
Especialización proyectos & 1 & $3 \%$ \\
Licenciatura finanzas & 1 & $3 \%$ \\
Licenciatura mediación pedagógica & 1 & $3 \%$ \\
N/R & 1 & $3 \%$ \\
Técnico salud ocupacional & 1 & $3 \%$ \\
& & $100 \%$ \\
\hline
\end{tabular}

Fuente: Instrumento aplicado

Elaboración: propia.

Se valora el manejo de idiomas como el inglés, portugués y lenguaje de señas costarricenses (LESCO) de la graduada, según se observa en los datos de la tabla 5:

\section{(c) (i) (9) (2)}

La Revista Estudios es editada por la Universidad de Costa Rica y se distribuye bajo una Licencia Creative Commons Atribución-NoComercial-CompartirIgual 3.0 Costa Rica. Para más información envíe un mensaje a revistaestudios.eeg@ucr.ac.cr. 
ISSN 1659-3316

Vasconcelos Vásquez Kattia Lizzett Montero Ulate Betzaida

Dossier: Mujeres y Educación en la Universidad Nacional

Red de Mujeres Investigadoras de la Universidad Nacional (UNA)

\section{Tabla 5}

Relación de nivel de conocimiento del idioma de la graduada de la carrera AGRH de la UTN

\begin{tabular}{cccc} 
Nivel/Idioma & Inglés & Portugués & LESCO \\
\hline Principiante & 21 & 29 & 27 \\
Básico & 26 & 0 & 3 \\
Intermedio & 28 & 1 & 5 \\
Avanzado & 6 & 1 & 0 \\
\hline Total & $\mathbf{8 1}$ & 31 & 35
\end{tabular}

Nota: Respuesta de opción múltiple

Fuente: Instrumento aplicado

Elaboración: propia.

El perfil socioeconómico de las graduadas de la carrera AGRH se caracteriza por diferentes condiciones que permiten el apoyo o respaldo económico para hacer frente a su ingreso, permanencia y conclusión de estudios universitarios, según se destaca en la tabla 6.

La Revista Estudios es editada por la Universidad de Costa Rica y se distribuye bajo una Licencia Creative Commons Atribución-NoComercial-CompartirIgual 3.0 Costa Rica. Para más información envíe un mensaje a revistaestudios.eeg@ucr.ac.cr. 
Dossier: Mujeres y Educación en la Universidad Nacional

Red de Mujeres Investigadoras de la Universidad Nacional (UNA)

Tabla 6

Apoyo socioeconómico de la graduada de la carrera AGRH de la UTN

\begin{tabular}{lcc}
\hline Curso/Especialización & $\begin{array}{c}\text { Frecuencia } \\
\text { absoluta }\end{array}$ & $\begin{array}{c}\text { Frecuencia } \\
\text { relativa }\end{array}$ \\
\hline Beca socioeconómica UTN & 51 & $47 \%$ \\
Ingresos propios & 24 & $22 \%$ \\
Ingresos familiares & 17 & $16 \%$ \\
Fonabe & 10 & $9 \%$ \\
Otros & 5 & $5 \%$ \\
Municipalidad & 1 & $1 \%$ \\
& & \\
\hline \multicolumn{1}{c}{ Total } & 108 & $100 \%$
\end{tabular}

Nota: Respuesta múltiple

Fuente: Instrumento aplicado

Elaboración: propia.

De las 51 graduadas que mencionan la asignación de una beca socioeconómica por parte de la UTN según la información descrita en la tabla 6, la distribución y composición de este beneficio se circunscribe, donde la beca 1 es la de menor nominación económica y la beca 5 contiene el reconocimiento máximo económico y condonación de créditos universitarios, según se especifica en la tabla 7.

\section{() $(\Theta \odot$}

La Revista Estudios es editada por la Universidad de Costa Rica y se distribuye bajo una Licencia Creative Commons Atribución-NoComercial-CompartirIgual 3.0 Costa Rica. Para más información envíe un mensaje a revistaestudios.eeg@ucr.ac.cr. 
Dossier: Mujeres y Educación en la Universidad Nacional

Red de Mujeres Investigadoras de la Universidad Nacional (UNA)

\section{Tabla 7}

Beca socioeconómica UTN de la graduada de la carrera AGRH

\begin{tabular}{|c|c|c|}
\hline Tipo Beca & $\begin{array}{c}\text { Frecuencia } \\
\text { absoluta }\end{array}$ & $\begin{array}{c}\text { Frecuencia } \\
\text { relativa }\end{array}$ \\
\hline Beca 5 & 34 & $51 \%$ \\
\hline Beca 1 & 16 & $24 \%$ \\
\hline Beca 4 & 15 & $22 \%$ \\
\hline Beca 2 & 1 & $1 \%$ \\
\hline Beca 1 & 1 & $1 \%$ \\
\hline
\end{tabular}

Fuente: Instrumento aplicado

Elaboración: propia.

Es de interés conocer la trayectoria profesional en cuanto a la ruta recorrida para su desarrollo profesional y laboral. Antes de esto se consulta a la graduada sobre el escenario provisto una vez finaliza su práctica profesional, información que se detalla en la figura 3:

\section{(c) (i)(2)}

La Revista Estudios es editada por la Universidad de Costa Rica y se distribuye bajo una Licencia Creative Commons Atribución-NoComercial-CompartirIgual 3.0 Costa Rica. Para más información envíe un mensaje a revistaestudios.eeg@ucr.ac.cr. 
Dossier: Mujeres y Educación en la Universidad Nacional

Red de Mujeres Investigadoras de la Universidad Nacional (UNA)

Figura 3

Empleabilidad posterior a la práctica profesional de la graduada de la carrera AGRH

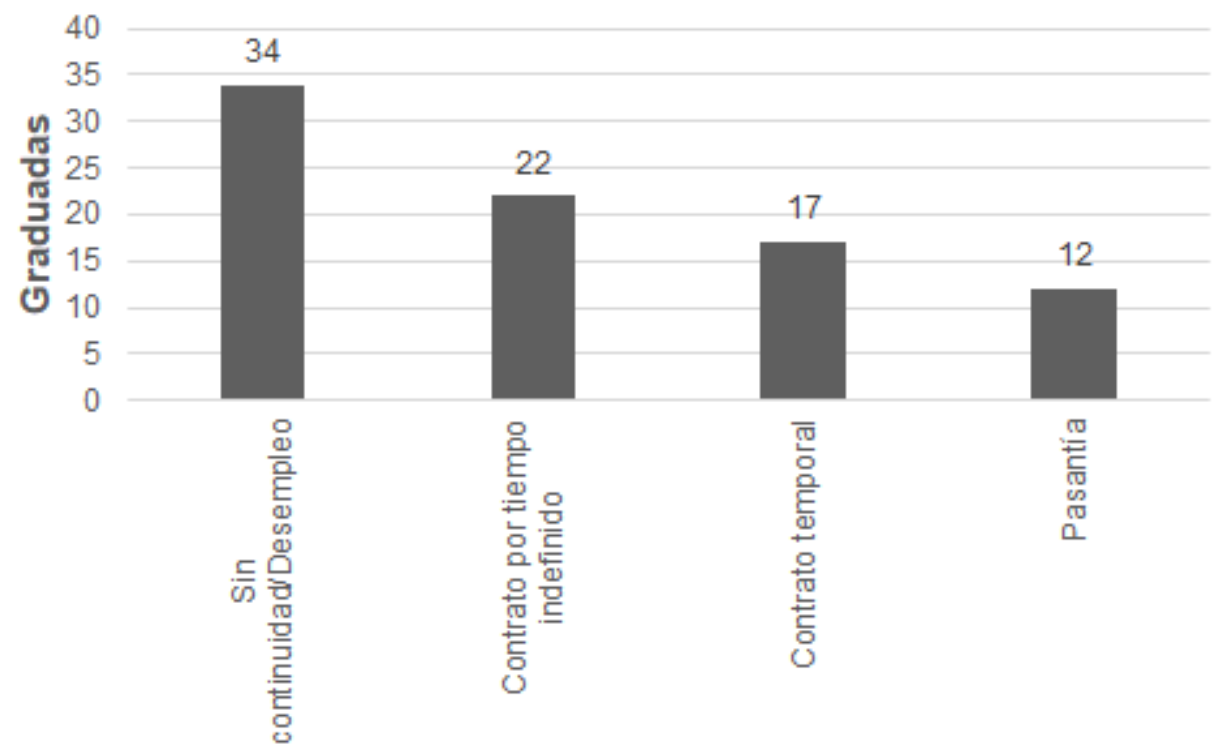

Fuente: Instrumento aplicado

Elaboración: propia.

Una de las características que conforman el perfil de la trayectoria laboral de la graduada está asociada con su experiencia laboral, observe la figura 4.

\section{(c) (i) (2) (2)}

La Revista Estudios es editada por la Universidad de Costa Rica y se distribuye bajo una Licencia Creative Commons Atribución-NoComercial-CompartirIgual 3.0 Costa Rica. Para más información envíe un mensaje a revistaestudios.eeg@ucr.ac.cr. 
Dossier: Mujeres y Educación en la Universidad Nacional

Red de Mujeres Investigadoras de la Universidad Nacional (UNA)

Figura 4

Años de experiencia laboral de la graduada de la carrera AGRH

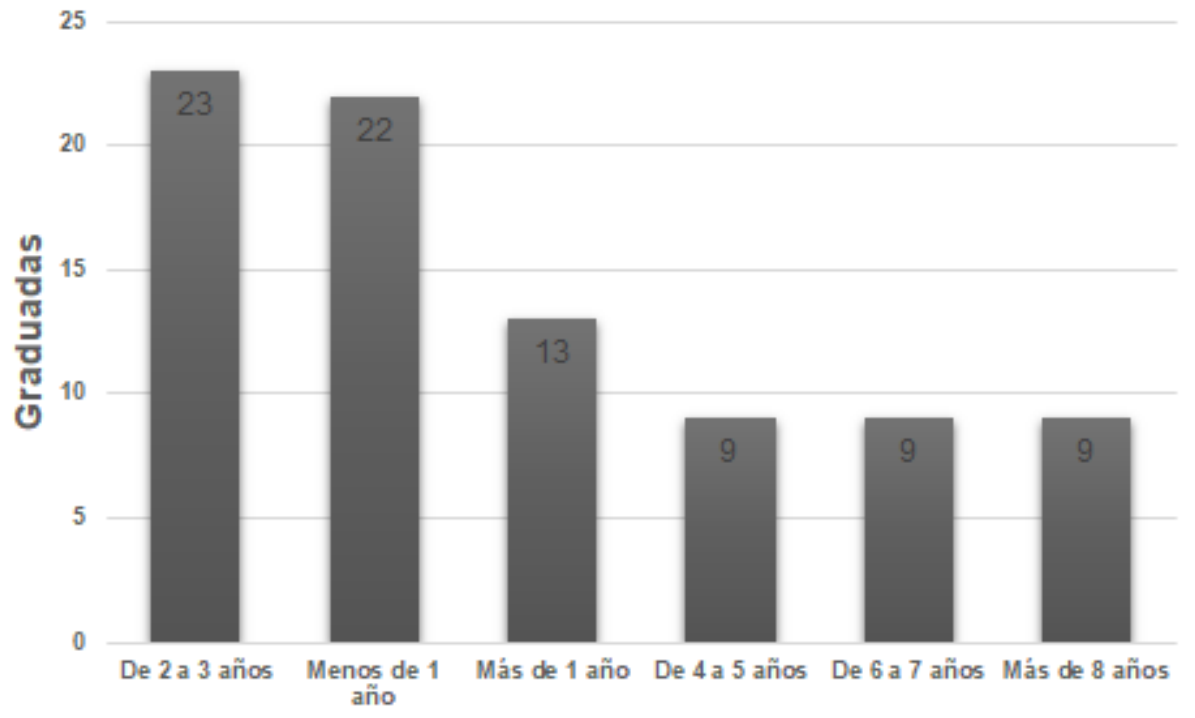

Fuente: Instrumento aplicado

Elaboración: propia.

El tipo de empresa y el grupo ocupacional que obtiene la graduada de la carrera AGRH completa el perfil de la trayectoria laboral de interés para el estudio, para ello ver la tabla 8.

\section{(c) $10(0)$}

La Revista Estudios es editada por la Universidad de Costa Rica y se distribuye bajo una Licencia Creative Commons Atribución-NoComercial-CompartirIgual 3.0 Costa Rica. Para más información envíe un mensaje a revistaestudios.eeg@ucr.ac.cr. 
Dossier: Mujeres y Educación en la Universidad Nacional

Red de Mujeres Investigadoras de la Universidad Nacional (UNA)

Tabla 8

Tipo de empresa en la que labora y grupo ocupacional de la graduada de la carrera AGRH de la UTN

\begin{tabular}{|l|c|l|c|}
\hline Tipo de Empresa & No. Graduadas & Grupo ocupacional & No. Graduadas \\
\hline Privada & 58 & Administrativo & 39 \\
Desempleo & 12 & Profesional & 14 \\
Pública & 10 & Desempleo & 13 \\
Emprendimiento & 4 & Operativo & 10 \\
ONG's & 1 & Técnico & 4 \\
& & Jefatura & 4 \\
& & Gerencia & 1 \\
\hline Total: & \multirow{25}{*}{} & & $\mathbf{8 5}$ \\
\hline
\end{tabular}

Fuente: Instrumento aplicado Elaboración: propia.

A partir de los resultados obtenidos se profundiza en el perfil profesiográfico para establecer sus características demográficas y definir la línea profesional de la graduada que es sujeto de migración para obtener su título universitario.

\section{Discusión}

Del estudio se derivan datos recopilados entre los meses de abril y mayo 2020 a las graduadas de la carrera AGRH de la UTN, y dado lo anterior, se hace posible la construcción del perfil profesiográfico a partir de elementos comunes en la población consultada.

\section{(c) $(\dot{0)} \otimes(2)$}

La Revista Estudios es editada por la Universidad de Costa Rica y se distribuye bajo una Licencia Creative Commons Atribución-NoComercial-CompartirIgual 3.0 Costa Rica. Para más información envíe un mensaje a revistaestudios.eeg@ucr.ac.cr. 
Dossier: Mujeres y Educación en la Universidad Nacional

Red de Mujeres Investigadoras de la Universidad Nacional (UNA)

\section{Perfil sociodemográfico:}

- La carrera AGRH de la UTN es la opción de educación universitaria para las mujeres, residentes en la provincia de Alajuela y sus cantones. Desde que se oferta la carrera y con la primera generación de bachillerato en noviembre 2012, se cuenta con una promoción de 409 graduadas que equivale a un $81 \%$ de los 503 profesionales juramentados en el período 2012 a 2019.

- La graduada de la carrera AGRH es una profesional joven, 50 de las 85 participantes se encuentran en un rango de edad de 20 a 27 años.

- Los cantones con mayor población de graduadas son el cantón central de Alajuela, que cuenta con 49 de las 85 participantes. Otros cantones de referencias son: Naranjo, Poás, Grecia, Palmares, Atenas, Sarchí y Orotina, lo que hace a la graduada una migrante universitaria.

- Con respecto al estado civil de la graduada, 58 de 85 participantes son solteras.

- La situación habitacional se destaca por las siguientes características: condición de casa de habitación para 60 estudiantes de 85 participantes que responden el documento, cuentan con una solución que les permite estabilidad desde el punto de vista que su techo es de sus progenitores 0 propio. Se destaca el número de personas que habitan y 69 de 85 participantes se clasifican en los rangos de 1 a 2 personas y de 3 a 4 personas, lo que supone que sus familias no superan los 4 miembros. Es relevante señalar que la graduada es responsable de uno o más personas dentro del núcleo familiar, para ello se consulta y 41 de 85 participantes indican que tienen una persona a cargo, lo cual se relaciona con un hijo (a) o alguno de sus progenitores.

\section{(C) $(\Theta \odot$}

La Revista Estudios es editada por la Universidad de Costa Rica y se distribuye bajo una Licencia Creative Commons Atribución-NoComercial-CompartirIgual 3.0 Costa Rica. Para más información envíe un mensaje a revistaestudios.eeg@ucr.ac.cr. 
Dossier: Mujeres y Educación en la Universidad Nacional

Red de Mujeres Investigadoras de la Universidad Nacional (UNA)

\section{Perfil académico:}

- La graduada de la carrera AGRH proviene de un colegio académico público y en este particular 61 de 85 participantes cumplen con esta condición.

- De acuerdo con el año de ingreso a la carrera y la obtención del título de bachillerato universitario, la graduada alcanza la meta en aproximadamente 4 años, a partir del 100\% de cumplimiento del programa de estudios. Es importante señalar en futuros espacios de investigación, si la duración de 4 años en la obtención del título tiene las mismas implicaciones en cuando al tiempo invertido o requerido para las graduadas en la franja diurna 0 nocturna.

- Se señala que 30 de 85 graduadas invierten en su actualización y desarrollo profesional. Lamentablemente, 55 graduadas se mantienen inactivas, sin embargo esta situación se convierte en una oportunidad para atraer a las graduadas a su alma mater a través de diversos programas que fortalezcan su pertenencia y actualización en la disciplina.

- De las graduadas activas en una especialización, curso técnico o certificación, se deriva que 20 graduadas se encuentran cursando una licenciatura. Cabe destacar la continuidad de las graduadas en el programa de licenciatura AGRH como la opción de mayor preferencia. Otras se destacan por cursos de inglés y contabilidad que son complementarios al quehacer del profesional de recursos humanos.

- Existe una oportunidad que debe atender de inmediato la graduada, en términos de alcanzar una mejoría significativa en el manejo de idiomas como el inglés y portugués. Es evidente que existe una brecha significativa que limita la posibilidad de desarrollo e incluso de inserción al mercado laboral, a 
Dossier: Mujeres y Educación en la Universidad Nacional

Red de Mujeres Investigadoras de la Universidad Nacional (UNA)

consecuencia de un nivel bajo entre principiante o básico en el conocimiento y manejo de los idiomas citados como competencia técnica.

- Solo 28 graduadas mencionan que se encuentran en un nivel de conocimiento intermedio para el idioma inglés. Esto les da una condición de B-2 según el marco común europeo de referencia, y a nivel de diversas industrias les hace más competitivas en términos del mercado laboral.

- El lenguaje de señas costarricenses (LESCO) como lengua viso-gestual es pretendida por los empleadores producto de iniciativas y programas de inclusión, por tanto, es una competencia técnica de interés para la graduada, $y$ es visto como un factor adicional para una futura oportunidad laboral en otras organizaciones.

\section{Apoyo socioeconómico:}

- La estudiante requirió del apoyo económico por parte de la UTN, que complementa con otras aristas para alcanzar su conclusión de estudios universitarios.

- La beca socioeconómica de la UTN es la fuente de financiamiento por excelencia que favorece a la egresada, a razón de que 67 de las 85 entrevistadas indican que se les asignó el beneficio de este rubro distribuido entre las cinco categorías que lo constituyen. Cabe destacar que 34 de las 67 graduadas beneficiarias contaron con la beca 5 que es la categoría máxima en términos económicos e incluye: apoyo monetario y otorgamiento de créditos a cero costos. En una proporción inferior se destaca la graduada que con recursos propios hace frente a los costos de créditos, materiales y

\section{(C) $(0 \odot$}

La Revista Estudios es editada por la Universidad de Costa Rica y se distribuye bajo una Licencia Creative Commons Atribución-NoComercial-CompartirIgual 3.0 Costa Rica. Para más información envíe un mensaje a revistaestudios.eeg@ucr.ac.cr. 
Dossier: Mujeres y Educación en la Universidad Nacional

Red de Mujeres Investigadoras de la Universidad Nacional (UNA) desplazamiento. Los núcleos familiares apoyan con una participación menor a este parámetro.

- En ausencia de un programa de becas en la UTN, las graduadas verían disminuidas sus posibilidades de titulación, lo que afectaría no solo su continuidad en la carrera, sino los plazos para el cumplimiento del plan de estudios sujeto a un tema de disponibilidad de recursos económicos, minimizando la posibilidad de inserción laboral o desarrollo profesional.

\section{Trayectoria profesional:}

- Se consulta a las entrevistadas una vez finalizada su práctica profesional como requisito para optar por el bachillerato universitario, cuáles son las condiciones o posibilidades de empleabilidad en relación con las empresas donde ejecutaron este proceso. Se destaca que 22 graduadas obtienen un contrato por tiempo indefinido y 17 un contrato temporal, lo que implica una inserción inmediata a la fuerza de trabajo. Asimismo, 12 de las graduadas participan en programas de pasantías, que les permite adquirir experiencia laboral en términos de su área de competencia y el proceso productivo de la organización. Caso contrario, 34 de las 85 graduadas no tienen continuidad, lo que contrasta a las graduadas AGRH con la cruda realidad del desempleo, que cada vez más aqueja al país, con mayor afectación a la población femenina en diferentes tipos de industrias.

- Los resultados muestran que 58 de las 85 graduadas cuentan con 3 años o menos de experiencia laboral. Esta condición de poca experiencia, sumado a las exigencias o requisitos que establecen los empleadores, ocupa a las graduadas en puestos de trabajo con funciones administrativas o incluso

\section{(C) $(0 \bigcirc$}

La Revista Estudios es editada por la Universidad de Costa Rica y se distribuye bajo una Licencia Creative Commons Atribución-NoComercial-CompartirIgual 3.0 Costa Rica. Para más información envíe un mensaje a revistaestudios.eeg@ucr.ac.cr. 
Dossier: Mujeres y Educación en la Universidad Nacional

Red de Mujeres Investigadoras de la Universidad Nacional (UNA)

operativas. Las graduadas con 40 más años de experiencia están representadas por 18 de 85 participantes, lo que las faculta a desempeñar posiciones a nivel técnico, profesional o incluso de jefatura, que permite afianzar un escenario que faculta a la graduada como la responsable de procesos técnicos que facilitan la toma de decisiones para los niveles superiores según lo que demanda las organizaciones con quienes tienen una relación laboral contractual.

- Cabe destacar que 58 de 85 graduadas actualmente se emplean en el sector privado. En el sector público se cuenta con 10 graduadas según el presente estudio. El autoempleo es una modalidad que motiva 4 graduadas que se encuentran en la modalidad de emprendimiento. Un total de 12 graduadas se encuentran desempleadas.

- El grupo ocupacional con mayor representatividad es el administrativo con 39 de las 85 graduadas. Se registran 14 graduadas que se desempeñan en puestos profesionales, 10 de ellas en posiciones operativas y 13 graduadas lamentable no cuenta con la posibilidad de un empleo.

\section{Perfil profesional de la graduada:}

- En cuanto al perfil de salida del profesional de la carrera AGRH y el comportamiento de la oferta y demanda del mercado laboral,

En cuanto al perfil de salida del profesional de la carrera AGRH, el comportamiento de la oferta y demanda del mercado laboral, se muestra que el $68 \%$ de las graduadas laboran actualmente para el sector privado. Un 12\% encuentra en el

\section{(c) (i) (9) (2)}

La Revista Estudios es editada por la Universidad de Costa Rica y se distribuye bajo una Licencia Creative Commons Atribución-NoComercial-CompartirIgual 3.0 Costa Rica. Para más información envíe un mensaje a 
Dossier: Mujeres y Educación en la Universidad Nacional

Red de Mujeres Investigadoras de la Universidad Nacional (UNA)

sector público la posibilidad de inserción a la fuerza laboral. Un $4 \%$ de las graduadas se emplean en sus propios emprendimientos y un $1 \%$ en organizaciones no gubernamentales (ONG). Además, el 14\% de la población en estudio se suma a la cifra de desempleo, del cual se hace referencia en párrafos anteriores.

\section{Algunas Conclusiones}

En síntesis, las graduadas de la carrera de AGRH se han formado en un área que les permite insertarse en el mercado laboral en diferentes sectores. Esto es muy positivo porque significa que la carrera no se limita a un solo campo. Para la gestión de la carrera es bueno considerar los puestos que están ocupando las graduadas y verificar el plan de estudios de manera que, en el próximo proceso de actualización, se amplíe y renueve el perfil de salida.

De los resultados se puede verificar que la visión y misión de la UTN se logra al ver que las graduadas están incorporándose en el mercado laboral como era esperado en el perfil de salida de la carrera. Por otra parte, la carrera de AGRH cumple con las demandas de los empleadores que conforman el mercado laboral y que requieren talento para diferentes grupos ocupacionales.

Un aspecto vital de la universidad pública es que hace que las personas logren sus sueños profesionales académicamente hablando, lo que sumado a la experiencia laboral le permite al profesional desarrollar una carrera desde puestos operativos y/o asistenciales, hasta puestos de trabajo con mayores demandas técnicas, responsabilidades o funciones que requieren mayor especialización, criterio, y autonomía para la toma de decisiones.

\section{(C) $(0 \odot$}

La Revista Estudios es editada por la Universidad de Costa Rica y se distribuye bajo una Licencia Creative Commons Atribución-NoComercial-CompartirIgual 3.0 Costa Rica. Para más información envíe un mensaje a 
Dossier: Mujeres y Educación en la Universidad Nacional

Red de Mujeres Investigadoras de la Universidad Nacional (UNA)

Para las investigadoras los resultados de esta investigación dan pie a sugerir otras líneas en las cuales se puedan obtener hallazgos diversos en cuanto a:

- Actualización de la carrera con la UTN.

- Análisis de la trayectoria profesional de las graduadas.

- Formación complementaria a la administración y gestión de recursos humanos.

- Mapa de carrera y trayectoria profesional.

- Actualización del plan de estudios de la carrera de AGRH.

- Análisis de la relación de las actividades desarrolladas por los profesionales de recursos humanos y los perfiles de la ocupación.

- Diferencias entre el perfil de salida de la carrera y el perfil de salida deseado o requerido por el mercado laboral.

- Perfil de competencias del profesional de recursos humanos.

Los resultados de este documento dan lugar a futuras líneas de investigación para la formulación de estudios de trayectoria académica, seguimiento a graduadas, opinión y demandas de empleadores, enriquecimiento del currículum, actualización del plan de estudios y tendencias del mercado laboral. Debe ser interés de la carrera identificar la línea base para proponer acciones de actualización en la disciplina que acompañen y permitan un avance de las graduadas en términos de su desarrollo y crecimiento laboral.

\section{(c) (i)(2)}

La Revista Estudios es editada por la Universidad de Costa Rica y se distribuye bajo una Licencia Creative Commons Atribución-NoComercial-Compartirlgual 3.0 Costa Rica. Para más información envíe un mensaje a 
Dossier: Mujeres y Educación en la Universidad Nacional

Red de Mujeres Investigadoras de la Universidad Nacional (UNA)

\section{Referencias.}

Borges Méndez R. (1995). El estudio de caso como instrumento pedagógico y de investigación en políticas públicas. Estudio de caso №.4 Universidad de Chile. Facultad de Ciencias Físicas y Matemáticas. Departamento de Ingeniería Industrial

Castro Monge, E. (2010). El estudio de casos como metodología de investigación y su importancia en la dirección y administración de empresas. Revista Nacional De Administración, 1(2), 31-54. Recuperado de https://doi.org/10.22458/rna.v1i2.332

Chetty, S. (1996) The Case Study Method for Research in Small- and Medium-sized Firms. International Small Business Journal 15(1), 73- 85.

Cohen, Néstor y Gómez Rojas G. (2019). Metodología, ¿para qué? La Producción de los datos y los diseños. Editorial Teseo, Red Latinoamericana de Metodología y CLACSO. p. 276. Buenos Aires.

Consejo Nacional de Rectores. (2019). Seguimiento de la condición laboral de las personas graduadas 2011-2013 de la Universidad Técnica Nacional. Oficina de Planificación de la Educación Superior. Observatorio Laboral de Profesiones.

Damián Simón, J., Arellano Mont, L. J. (2009). Calidad profesional del técnico superior universitario en administración. Una visión de graduados y de empleadores. Actualidades Investigativas en Educación, 9(2), 7-8. Recuperado de https://biblat.unam.mx/hevila/Actualidadesinvestigativaseneducacion/2009/v ol9/no2/6.pdf

Gómez Rojas Gabriela y A. De Sena (comp.) (2019). Buenos Aires: Ediciones Cooperativas. http://biblioteca.clacso.edu.ar/clacso/se/20190823024606/Metodologia para que.pdf

\footnotetext{
(U⿱宀一)

La Revista Estudios es editada por la Universidad de Costa Rica y se distribuye bajo una Licencia Creative Commons Atribución-NoComercial-CompartirIgual 3.0 Costa Rica. Para más información envíe un mensaje a revistaestudios.eeg@ucr.ac.cr.
} 
Dossier: Mujeres y Educación en la Universidad Nacional

Red de Mujeres Investigadoras de la Universidad Nacional (UNA)

Núñez Masís, L. C. y Marín Alfaro, A. (2018). La profesión de Turismo Ecológico en la Universidad de Costa Rica: Un análisis desde la perspectiva de su población graduada. Revista Educación, 42 (2), 5-6. Recuperado de https://www.scielo.sa.cr/pdf/edu/v42n2/2215-2644-edu-42-02-00184.pdf

Pérez Aguilar, W. (1999). El estudio de casos en Metodología para la investigación en marketing y dirección de empresas. En F.J. Sarabia A. (ed), Piramide Madrid, p. 2321.

Plan de Estudio Bachillerato en Administración y Gestión de Recursos Humanos (2011). Carrera AGRH, Universidad Técnica Nacional, 27-28.

Plan General de Investigación (2017-2021). Área de Ciencias Administrativas. Bachillerato y Licenciatura en Administración y Gestión de Recursos Humanos con salida lateral al Diplomado de Administración de Recursos Humanos.

Stake, R. (2010). Qualitative Research Studying How Things Work . The guiford Press, p. 244.

Universidad Técnica Nacional (2020-05-05). Informe de labores y rendición de cuentas 2019 Resumen Ejecutivo. Administración Universitaria. Dirección de Comunicación Institucional. Recuperado de www.utn.ac.cr/utn-transparente

Universidad Técnica Nacional (2020-05-26). Plan de Estudios Carrera AGRH. Recuperado de http://utn.ac.cr/content/administraci\%C3\%B3n-ygesti\%C3\%B3n-de-recursos-humanos

Universidad Técnica Nacional (2020-07-15). Política de Admisión. Recuperado de http://utn.ac.cr/sites/default/files/attachments/Poli\%CC\%81ticas\%20de\%20a dmisio\%CC\%81n 0.pdf

Vacilachis I. Ameigeiras A., Chernobilsky L., Gimenez V., Mallimaci F. Mendizabal N., Neiman G., Quaranta G. y Soneira A. (2006). Estrategia de Investigación Cualitativa. Barcelona: Gedisa.

Yin, R. K. (1981). The Case Study Crisis: Some Answers. Administrative Science Quarterly, $26(1)$, 58-65.

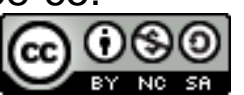

La Revista Estudios es editada por la Universidad de Costa Rica y se distribuye bajo una Licencia Creative Commons Atribución-NoComercial-CompartirIgual 3.0 Costa Rica. Para más información envíe un mensaje a revistaestudios.eeg@ucr.ac.cr. 
Dossier: Mujeres y Educación en la Universidad Nacional

Red de Mujeres Investigadoras de la Universidad Nacional (UNA)

Yin, R. K. (1989). Case Study Research. Design and Methods, Sage, Cuarta Impresión, Ed. Newbury Park, CA.

Yin, R. K. (1993). Applications of Case Study Research, Sage, Ed. Newbury Park, CA [¿cuál es el nombre de la editorial?]

Yin, R. K. (1999) Enhancing the quality of Case Studies in Health Services Research. Health Services Research, 34 (5), 1209- 1224. 